\title{
BRAF V600E mutation correlates with aggressive clinico- pathological features but does not influence tumor recurrence in papillary thyroid carcinoma-10-year single-center results
}

\author{
Navid Tabriz ${ }^{1}$, Johannes Grone ${ }^{1}$, Verena Uslar ${ }^{1}$, Andrea Tannapfel ${ }^{2}$, Dirk Weyhe $^{1}$ \\ ${ }^{1}$ University Hospital for Visceral Surgery, Pius Hospital Oldenburg, Medical Campus University of Oldenburg, Oldenburg, Germany; ${ }^{2}$ Institute of \\ Pathology, Ruhr-University Bochum, Bochum, Germany \\ Contributions: (I) Conception and design: N Tabriz, J Grone, A Tannapfel, D Weyhe; (II) Administrative support: V Uslar, N Tabriz; (III) Provision \\ of study materials or patients: D Weyhe, N Tabriz, J Grone; (IV) Collection and assembly of data: J Grone; (V) Data analysis and interpretation: J \\ Grone, V Uslar; (VI) Manuscript writing: All authors; (VII) Final approval of manuscript: All authors. \\ Correspondence to: Dr. Navid Tabriz. Universitätsklinik für Viszeralchirurgie, Pius-Hospital Oldenburg, Georgstr. 12, 26121 Oldenburg, Germany. \\ Email: navid.tabriz@uol.de.
}

\begin{abstract}
Backgrounda BRAF V600E mutation is common in papillary thyroid carcinoma (PTC) but its prognostic value and influence on tumor recurrence is controversial. We investigated if BRAF V600E mutation influences tumor behavior and recurrence, and if it can be used as surrogate parameter in PTC.

Methods: In a single center retrospective study with a median follow-up of 5 years, incidence of BRAF V600E mutation in 186 PTC specimens from 2007-2016 was investigated. Tumor outcome parameters including TNM status, multifocal and invasive growth and tumor recurrence rate were examined.

Results: In 98 specimens (52.7\%) a BRAF V600E mutation (BRAF+), and in 88 specimens (47.3\%) no mutation (BRAF-) was detected. There was no gender specific difference. BRAF+ patients were significantly older (mean 5.6 years; $\mathrm{P}=0.011$ ). BRAF+ tumors were significantly smaller (14.4 vs. $18.3 \mathrm{~mm} ; \mathrm{P}=0.018$ ), and more often showed a multifocal $(30.6 \%$ vs. $17 \% ; \mathrm{P}=0.031)$ and extracapsular tumor growth pattern (pT3b and pT4a; BRAF+ 22.4\% vs. BRAF- 10.2\%; $\mathrm{P}=0.026)$. Although lymph node-status did not differ in both groups, BRAF+ showed a higher infiltration rate of the lateral lymph node compartment $(12.2 \%$ vs. $5.7 \%$; $\mathrm{P}=$ n.s.). Distant metastases occurred only in BRAF+ (3.1\% vs. $0 \%)$. There was no significant difference in terms of tumor recurrence rate.

Conclusions: Results regarding the incidence of malignant lymph nodes, tumor growth pattern and tumor multifocality suggest a more aggressive tumor behavior in BRAF+ PTC but this fact does not affect tumor recurrence rate in a five year follow up period. Therefore, the postoperative role of BRAF V600E mutation remains unclear, and a general change of operative procedure and radicality cannot be recommended based on BRAF status alone.
\end{abstract}

Keywords: Papillary thyroid carcinoma (PTC); multifocal tumor growth; extrathyroidal tumor growth; recurrence rate; tumor aggressiveness; BRAF V600E

Submitted Feb 19, 2020. Accepted for publication Jul 09, 2020.

doi: $10.21037 /$ gs-20-244

View this article at: http://dx.doi.org/10.21037/gs-20-244

\section{Introduction}

The incidence of thyroid carcinoma has increased worldwide in recent years, with papillary thyroid carcinoma (PTC) representing the highest proportion $(1,2)$.
In adults, women are affected about four times more frequently than men. Although PTC has an excellent prognosis, local lymph node metastases, especially in the central compartment (level 6), are identified in about $60 \%$ 
of cases $(3,4)$. These lymph node metastases correlate with development of locoregional tumor recurrence resulting in repeated treatment (5).

The surgical therapy of PTC depends on tumor and lymph node status and even differs between continents. The American and European guidelines recommend a total or near total thyroidectomy for PTCs, with a more limited resection only for low-risk tumors (microcarcinoma). In Japan, in case of papillary microcarcinoma an active surveillance with close monitoring without a resection can be recommended in certain cases (6-8). The implementation of a central neck dissection in nodal-positive PTC is generally accepted but a prophylactic lymphadenectomy in clinical nodal-negative cases remains controversial due to increased risk of complications $(9,10)$.

However, the diagnostic options for preoperative assessment of lymph node metastasis, especially in the central compartment, are very limited. Therefore, the stratification for a possibly more aggressive tumor course is based on postoperative histopathological markers (i.e., extrathyroidal extension, larger tumors) $(11,12)$. In recent years, molecular markers have been examined to establish a risk stratification in order to detect potentially more aggressive tumors and to offer a more individualized surgical approach. Among these markers, BRAF V600E (in the following abbreviated as BRAF) has received the widest interest. In PTCs, BRAF mutation is the most common genetic change with $30 \%$ to $90 \%(45-80 \%$ in the conventional variant, $5-25 \%$ in the follicular variant, $60-95 \%$ in the tall-cell variant) (13-15).

BRAF V600E mutation promotes tumorigenesis by activating the MAP kinase pathway leading to an increase in cell growth, proliferation and differentiation (16). Many studies have demonstrated that BRAF mutation status is associated with aggressive tumor features such as capsule invasion, extrathyroidal extension and lymph node metastasis, and that it can increase the risk of persistent and recurrent disease (17). Furthermore, some studies recommend a more radical surgical approach in cases of preoperatively confirmed BRAF mutation in fine needle aspiration $(18,19)$. However, several studies have failed to confirm these findings, leaving the overall significance of the BRAF mutation unclear (20-23). Therefore, the role of BRAF regarding tumor relapse still remains controversial, and standardized BRAF analysis has therefore not yet been included in the relevant guidelines.

This single-centre retrospective study was conducted to investigate the influence of BRAF mutation on tumor course, tumor recurrence and patients' outcome in PTC from 2007 to 2016. We present the following article in accordance with the STROBE reporting checklist (available at http://dx.doi.org/10.21037/gs-20-244).

\section{Methods}

The study was conducted in accordance with the Declaration of Helsinki (as revised in 2013). The study was approved by the institutional/regional/national ethics/ committee/ethics board of the Carl von University of Oldenburg (No. 005/2017) and informed consent was taken from all the patients.

\section{Patients}

Between 2007 and $2016 \mathrm{n}=382$ patients with thyroid cancer were treated in the University Clinic for Visceral Surgery, Pius-Hospital, Medical Campus University of Oldenburg; 285 cases of PTC were detected and 270 patients were contacted for permission to determine the mutation status for BRAF mutation (BRAF positive or short BRAF+ vs. BRAF negative or short BRAF-). The written consent of 189 patients could be obtained (Figure 1). Written consent was needed, because BRAF analysis exceeded the typical diagnostics, and because of handling specimen containing genetic material.

The following data was gathered for all patients for whom consent was obtained: Preoperative TSH (serum thyrotropin), preoperative sonographic features, the amount of postoperative radioiodine therapy (RIA), tumor recurrence and characteristics, and time of re-operation for tumor recurrence were noted.

Postoperative complications, i.e., recurrent nerve paralysis, hypocalcemia, and wound infection were recorded prospectively as part of the quality assurance measures in our thyroid center. As the TNM-classification has changed during the last 10 years, the TNM status was adopted to the actual $8^{\text {th }}$ edition (24).

\section{DNA extraction/amplification and BRAF-detection}

Part of the paraffin-embedded tissue was prepared for microscopy, and the areas with carcinoma were marked on the microscope slides. Thus, a small part of the tumor could be removed from the paraffin block. The gathered DNA was cleaned and fluorescence measurements for determining of the DNA concentration were carried out with a 


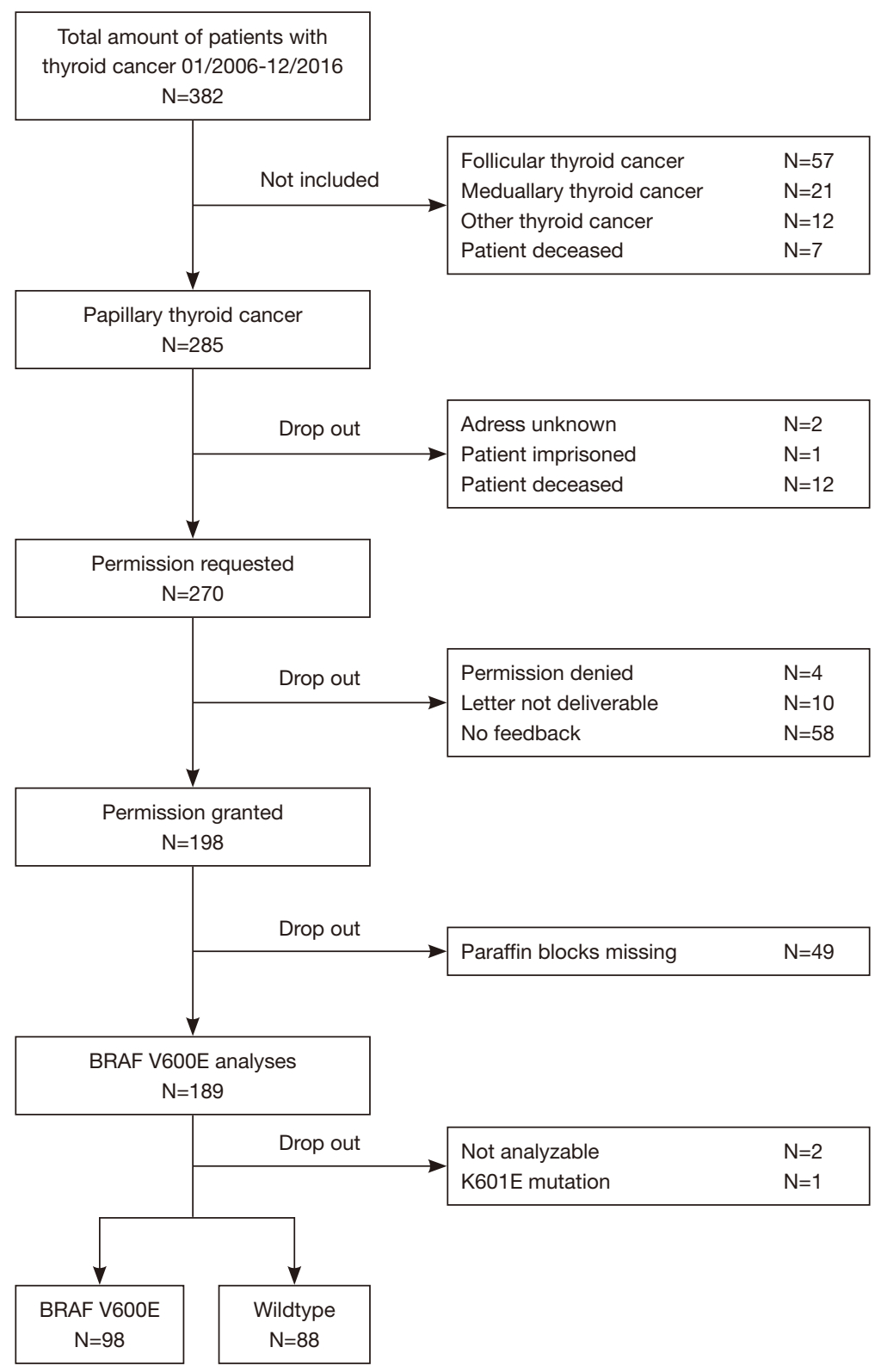

Figure 1 Flow chart depicting inclusion and exclusion criteria.

spectrophotometer. DNA amplification was done by PCR (polymerase chain reaction) followed by pyrosequencing for determination of the mutation status as already published $(25,26)$.

In summary, the genomic DNA was amplified by standard PCR using the forward primer "5-TCCTTTACTTACTAC ACCTCAGAT-3" and the reverse primer "3-CCCACTCC ATCGAGATT-5". The forward pyrosequencing primer was 5'-TGATTTTGGTCTAGCTACA-3', and pyrosequencing was performed using PyroMark ${ }^{\circledR}$ Q24 System (Qiagen).

\section{Statistical analysis}

Data collection was performed using Microsoft Excel, Version 2007 Professional. For statistical analysis the program IBM SPSS Statistic 25 was used. Continuous data was described by calculating mean, standard deviation, 
median, minimum, and maximum. The differences in patient characteristics between the BRAF+ and the BRAFgroup where analyzed as follows: categorically scaled features with $\leq 4$ characteristic values were tested for independence using non-parametric testing. By default, the Pearson chi-square independence test was used. At an expected frequency $<5$, Fisher's Exact Test was used instead. For categorically scaled characteristics with $>4$ feature values, risk ratio with $95 \%$ confidence interval was calculated. No tests were performed for variables describing patient characteristics irrelevant to our research question in order to keep the number of tests low. We corrected for multiple testing using the Benjamini-Hochberg method. Metric variables included age, preoperative TSH, sonographic nodule diameter, and thyroid volume, pathologic tumor size, TNM staging. Categorically scaled variables included gender, (extrathyroid) tumor expansion, (multifocal) tumor growth, pathologic variant, lymph node metastasis, distant metastasis and BRAF mutation status. Factors favoring development of recurrence were examined by means of multiple logistic regressions with forward and backward selection. For this analysis the program JASP was used.

\section{Results}

\section{Tumor characteristics}

A total of 189 tumor specimens were subjected to BRAF mutation analysis (Figure 1). Out of these samples, two cases could not be analyzed even with repeated pyrosequencing and one patient showed a $\mathrm{K} 601 \mathrm{E}$ mutation, therefore these three cases were excluded and eventually a number of 186 specimens were analyzed. $\mathrm{N}=98(52.7 \%)$ specimens were $\mathrm{BRAF}+$ and $\mathrm{n}=88$ (47.3\%) were BRAF-.

Patient and tumor characteristics are illustrated in Table 1. The mean follow-up time was 5 years for both groups. There was no significant difference in gender distribution between the groups but a tendency for male sex in the $\mathrm{BRAF}+$ group. $\mathrm{BRAF}+$ patients were significantly older (5.6 years) at time of diagnosis $(\mathrm{P}<0.011)$. Preoperative thyrotropin $(\mathrm{TSH})$ levels did not differ between the groups.

Despite the significantly smaller tumor size in the BRAF+ group (14.4 vs. $18.3 \mathrm{~mm} ; \mathrm{P}=0.018), \mathrm{BRAF}+$ tumors showed a multifocal growth pattern $(31.6 \%$ vs. $17.9 \% ; \mathrm{P}=0.031)$ and a higher rate of extrathyroidal growth, $\mathrm{pT} 3 \mathrm{~b} / \mathrm{pT} 4 \mathrm{a}$ (22.4\% vs. $10.2 \% ; \mathrm{P}=0.026$ ). Although the total number of lymph node metastases was comparable in both groups, the
$\mathrm{BRAF}+$ group showed a higher infiltration rate of the lateral lymph node compartment (12.2\% vs. $5.7 \%$; n.s.). In case of microcarcinoma after hemithyroidectomy, a total resection including the contralateral thyroid tissue plus prophylactic central node dissection was only performed if thyroid nodules were existing in the remnant tissue. Patients with pT1b and more advanced carcinoma were treated by total thyroidectomy and prophylactic central node dissection. An additional ipsilateral or contralateral lymph node dissection was only performed in case of macroscopic proof of potential metastasis. As expected, the rate of remote metastasis was very low in both groups. However, distant metastases occurred only in the BRAF+ group $(3.1 \% v s$. $0 \%$; n.s.).

BRAF mutation was significantly prevalent in the classic tumor subtype (79\% vs. $47 \%$ ) and less in the follicular variant of $\mathrm{PTC}(17 \%$ vs. $47 \%$ in $\mathrm{BRAF}-\mathrm{P}<0.001)$. The ageindependent summary of the TNM classification according to the actual $8^{\text {th }}$ edition showed that $99 \%$ of BRAF- patients could be assigned to stage 1 . By contrast, $14.2 \%$ of the patients were allocated of stage 2 and higher in BRAF+. However, age also played a crucial role as $n=11,32.4 \%$ of the patients older than 55 years were assigned to stage 2 .

\section{Tumor therapy}

A total of 173 total thyroidectomies and 13 hemithyroidectomies were performed. No BRAF dependent significant difference were detected $(\mathrm{P}>0.6)$. Also, with regard to the implementation and extension of lymph node dissection no significant differences between the groups could be revealed.

An ablative RIA was performed in 168 cases (90.3\%) and 89 patients $(47.8 \%)$ were treated with more than one therapy.

Concerning perioperative complications (laryngeal nerve palsy, hypocalcemia and re-operation due to infection or bleeding) no significant differences between the groups could be found. It should be mentioned that due to the retrospective character of this study a differentiation between persistent and transient laryngeal nerve palsy and an information to postoperative hypoparathyroidism due to lack of routinely determined parathormone values cannot be made.

\section{Microcarcinoma}

A total of $n=63(n=34$ BRAF + and $n=29$ BRAF-) 
Table 1 patient and tumor characteristics for all patients included in the study and stratified by BRAF V600E-status

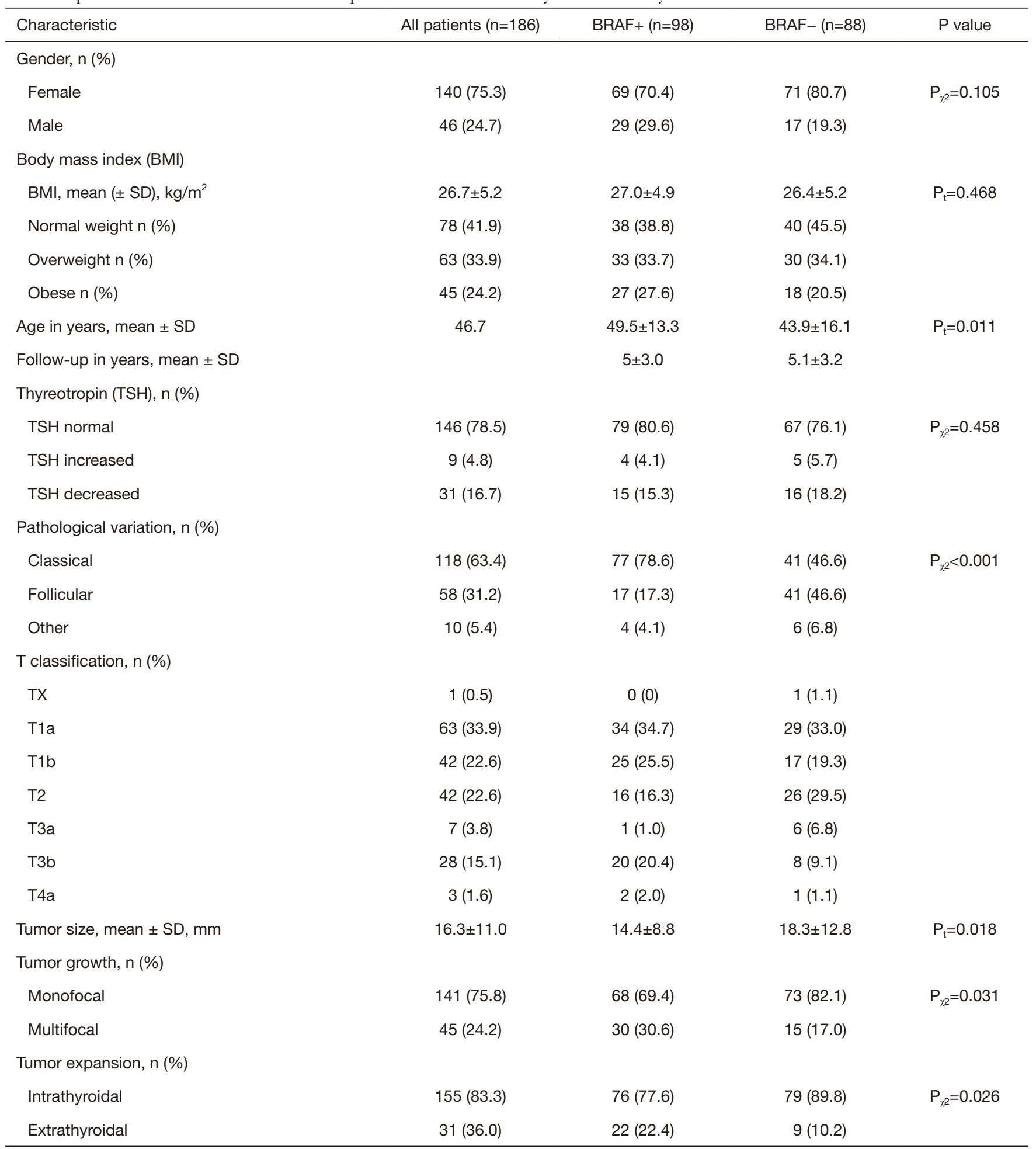

Table 1 (continued) 
Table 1 (continued)

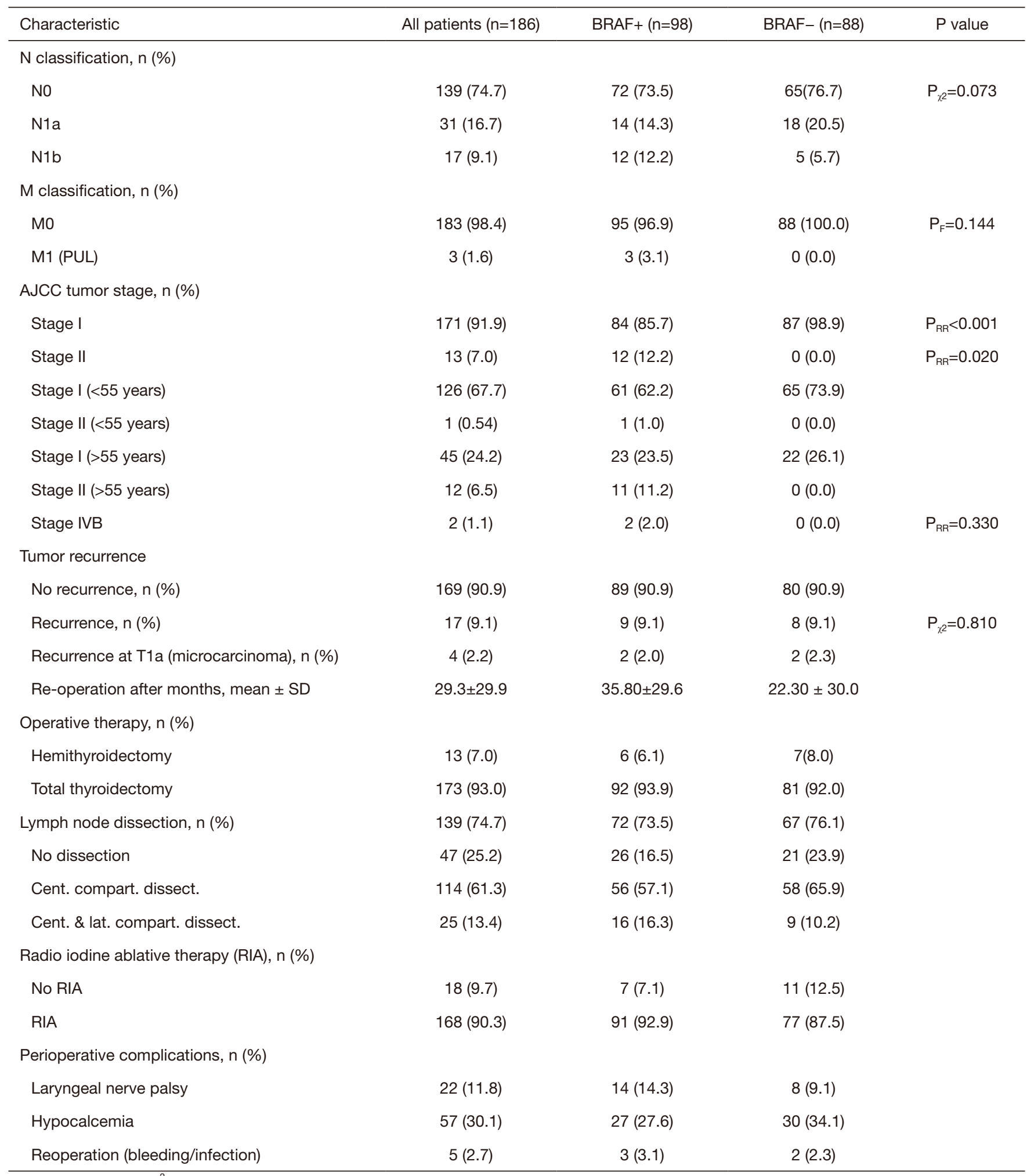

$\mathrm{P}_{\mathrm{t}}=$ t-test; $\mathrm{P}_{\mathrm{x} 2}=\mathrm{Chi}^{2}$-test; $\mathrm{P}_{\mathrm{F}}=$ Fisher's test; $\mathrm{P}_{\mathrm{RR}}=$ risk ratio. 


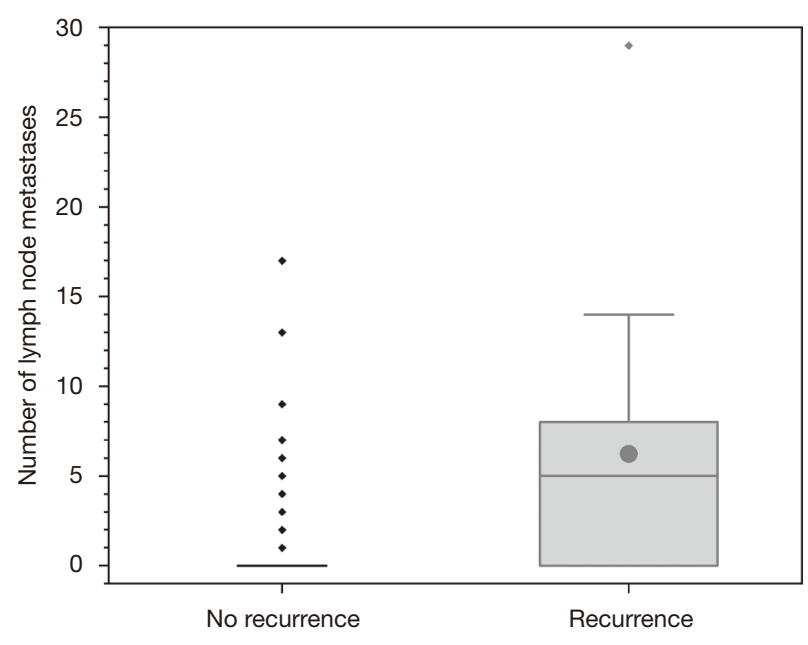

Figure 2 Number of lymph node metastases at the time of primary surgery for patients who later develop a relapse and for patients who do not develop a relapse in the follow-up period. The box depicts the upper and lower quartile, the vertical lines the median and the filled dots the mean. Whiskers indicate 1.5 times the interquartile range.

microcarcinoma ( $\mathrm{pT} 1 \mathrm{acN} 0)$ were removed in the examined period of which $n=9(n=3 B R A F+, n=6 B R A F-)$ were resected during hemithyroidectomy. A total of $n=4$ ( 2 in each group BRAF+) were re-operated because of lymph node metastasis. None of the patients who previously underwent hemithyroidectomy required reoperation.

\section{Tumor recurrence}

Tumor recurrence was defined as any positive disease found on the whole-body scan in a previously negative area with a correlating positive finding seen on clinical imaging (e.g., neck ultrasound or CT scan) that could be approached surgically. Metastatic patients were also coded as recurrent at time of diagnosis. In 17 individuals $(\mathrm{n}=9 \mathrm{BRAF}+$ and $\mathrm{n}=8$ BRAF-; n.s.) a recurrence of the PTC was detected in a mean follow-up period of five years. Of the individuals without lymph node metastasis $(\mathrm{n}=139)$ at time of primary treatment, $\mathrm{n}=5(5.1 \%)$ patients developed a recurrence. In patients with initial central lymph node metastasis $(n=31)$ and in individuals with lateral lymph node metastasis $(\mathrm{n}=17)$ tumor recurrence occurred in $5(16.1 \%)$ and $4(23.5 \%)$ cases, respectively. In addition, the number of lymph node metastasis at time of initial operation was higher in patients who developed tumor recurrence (mean 6.2) later, than in patients without tumor recurrence (mean 1.3; Figure 2).
Out of the patients with distant metastasis $(n=3)$ which all occurred in the lung, $\mathrm{n}=2$ patients also developed a local recurrence. In one patient only the lung metastasis could be detected on the body scan without local neck recurrence. Regarding the recurrence rate and the recurrence timepoint defined as time of re-operation after primary diagnosis no significant difference between BRAF + and BRAFpatients was found $(\mathrm{P}>0.05)$.

To further determine variables effecting tumor recurrence, a multiple logistic regression model with iterative backward and forward selection containing the following parameters was performed: age at surgery, body mass index, sex, tumor variant, tumor focality, tumor extension, pathologic tumor size, information about the number of lymph node metastasis $(0,<6$, or $\geq 6)$, distant metastasis, and BRAF mutation status. A model containing the information about the number of lymph node metastases, and distant metastasis can best explain tumor recurrence according to the area under the curve (AUC $=0.796$ ) with a sensitivity of 0.111 , and a specificity of 0.994 $\left[\mathrm{Chi}^{2}(183)=27.992, \mathrm{P}<0.001\right]$. Adding the BRAF mutation to this model, the occurrence of relapse increases sensitivity to 0.389 , and decreases specificity slightly to 0.976 , while keeping the AUC nearly constant $\left[\mathrm{AUC}=0.792 ; \mathrm{Chi}^{2}(182)\right.$ $=28.267, \mathrm{P}<0.001]$. However, BRAF status alone is not a significant influencing factor in this model (see Table 2).

\section{Discussion}

Due to the excellent course of PTC, the prognosis is influenced by disease-free survival rather than the overall survival unlike other types of cancer with higher mortality. Approximately $30 \%$ of patients with differentiated thyroid carcinoma suffer from tumor persistence or recurrence (27). Therefore, the focus is on precise detection of these patient groups for an individualized therapy and follow-up. In recent years, the BRAF V600E mutation is regarded as a surrogate marker for a more aggressive tumor behavior, but its influence on tumor recurrence remains controversial as many studies have failed to prove correlations of BRAF status and tumor outcome $(22,28,29)$.

In our study, $52.7 \%$ of the examined tumors were $\mathrm{BRAF}+$. This patient group was significantly older than the BRAF wild-type group irrespective of tumor stage. In fact, age has been already described as a risk factor in thyroid cancer in general (6). Regarding the cut-off point of 55 years which demarks the age associated risk in thyroid cancer, BRAF status significantly influences tumor progression in 
Table 2 Results of the multiple logistic regression: (I) best predictive model, (II) model including BRAF status

\begin{tabular}{|c|c|c|c|c|c|c|c|c|}
\hline \multirow{2}{*}{ Variables } & \multirow{2}{*}{ Estimate } & \multirow{2}{*}{$\begin{array}{l}\text { Standard } \\
\text { error }\end{array}$} & \multirow{2}{*}{$\begin{array}{l}\text { Odds } \\
\text { ratio }\end{array}$} & \multirow{2}{*}{ z-value } & \multicolumn{2}{|c|}{ Wald test } & \multicolumn{2}{|c|}{$\begin{array}{l}95 \% \text { confidence interval } \\
\text { (odds ratio scale) }\end{array}$} \\
\hline & & & & & Wald statistic & $P$ value & Lower bound & Upper bound \\
\hline (Intercept) & -3.384 & 0.441 & 0.034 & -7.669 & 58.810 & $<0.001$ & 0.014 & 0.081 \\
\hline \multicolumn{9}{|c|}{ (II) Coefficients if BRAF status is included } \\
\hline (Intercept) & -3.230 & 0.518 & 0.040 & -6.237 & 38.905 & $<0.001$ & 0.014 & 0.109 \\
\hline LN information* & 1.621 & 0.358 & 5.059 & 4.531 & 20.529 & $<0.001$ & 2.509 & 10.202 \\
\hline
\end{tabular}

${ }^{*}$, LN information coded as: number of lymph node metastasis $(0,<6$, or $\geq 6)$. ${ }^{\star \star}$, PM status: distant metastasis present (yes/no). ${ }^{* \star \star}$, BRAF status: BRAF positive/negative.

this patient group. Recently, it has been demonstrated that age-associated mortality risk is dependent on BRAF status, and therefore it has been suggested to differentiate between patients with BRAF V600E mutation and wild-type when applying age to risk stratification and management of PTC (30).

Despite of smaller tumor size, a multifocal, and a more extensive extrathyroidal tumor extension were detected in BRAF+ patients. Although the amount of lymph node metastases did not differ between the two groups, BRAF+ tumors affected the lateral lymph node compartment twice as often, and distant metastases were only seen in the $\mathrm{BRAF}+$ group. These results are in line with the mentioned studies suggesting a more aggressive tumor behavior of BRAF+ PTC (see Table 3).

A major weakness of retrospective analyses relates to the definition of the term "tumor recurrence" as the differentiation between persistent and recurrent disease is difficult due to the fact that routine follow-ups occur every 6 to 12 months, and a differentiation between recurrence and persistence as defined in the classic oncological definition is not always possible. Moreover, it is assumed that in most cases tumor recurrence in thyroid cancer is rather tumor persistence than true tumor recurrence due to incomplete preoperative staging and/or incomplete surgery (40).

Therefore, we used the term tumor recurrence defined as "the reappearance of tumor (either locally in thyroid bed, in neck nodes, or as distant metastatic disease) after a well-documented disease-free period" as proposed by Elisei et al. (41). Hence, we could exclude persistent tumor diseases and exclusively analyze patients with "real" recurrence. Irrespective of BRAF-status, we observed a tumor recurrence rate of $9.1 \%$ which did not differ between the groups, and we also did not observe differences regarding microcarcinoma incidences.

The largest cohort study to date showed that BRAF mutation is an independent risk factor for tumor recurrence over a median follow-up of 36 months (17). But in this study the overall recurrence rate was fairly high, regardless of the mutation status (20.9\% in BRAF+, $11.6 \%$ in BRAF-). This is probably due to the fact that biochemical and macroscopic recurrences have been summarized. NiedererWüst et al. detected a 10 -year recurrence-free survival in their retrospective single-center cohort of patients with PTC larger than $1 \mathrm{~cm}$ of $94 \%$ which fits with our data. Interestingly, in their analysis BRAF V600E mutation status was not associated with clinicopathologic characteristics of aggressive behavior such as extrathyroidal extension, lymph node metastases, higher T-categories, male sex, and older age (42). Due to our study design, we could not calculate survival time or disease-free survival, as we could only include patients who were still alive due to the consent required to analyze the pathological samples. This increases the uncertainty of the respective analysis to such an extent that it would be essentially meaningless. However, since the median follow-up of both groups is similar, and since 
Table 3 Relationship between BRAF V600E mutation and potential prognostic factors in selected studies

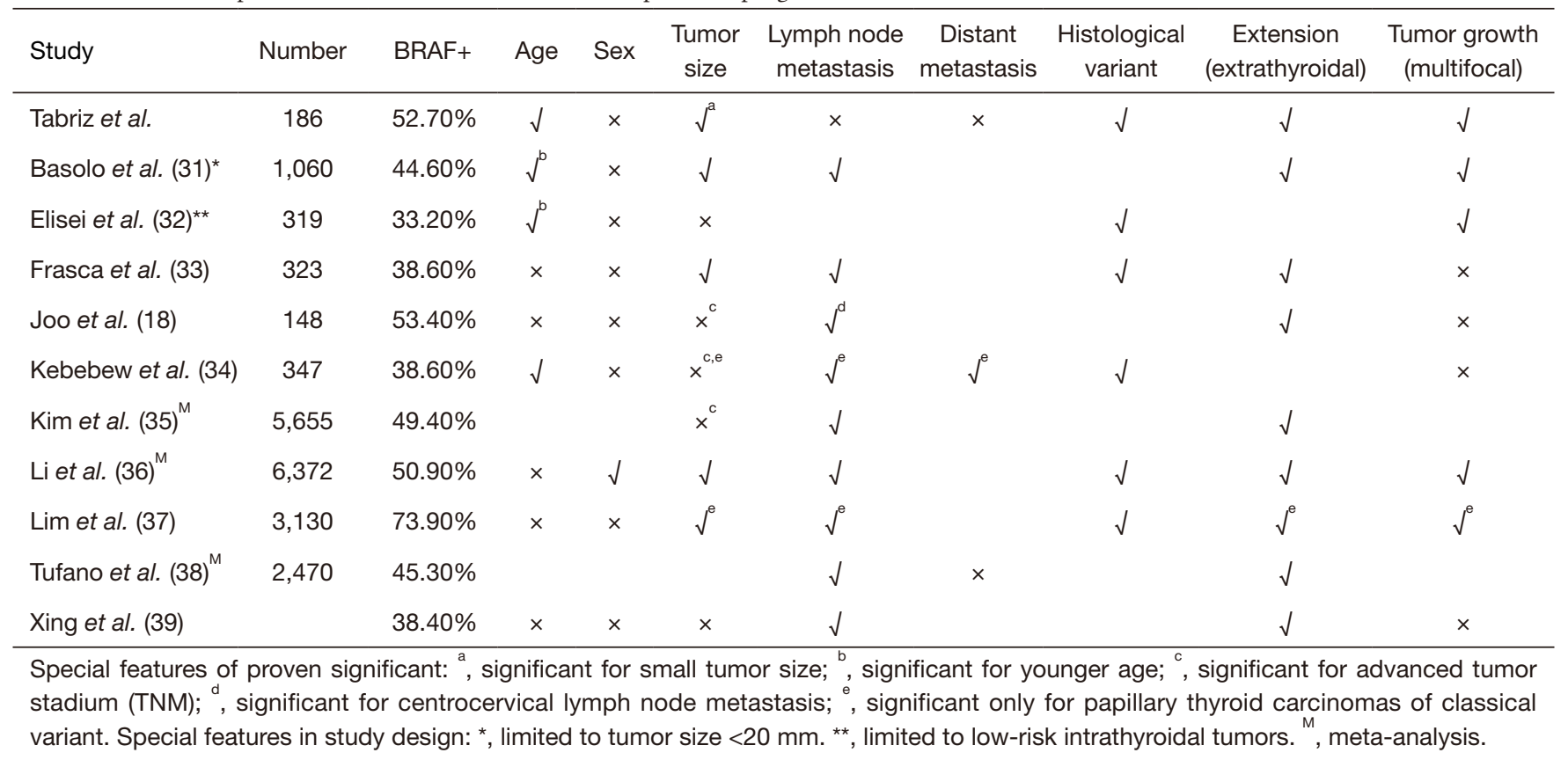

the size of the $\mathrm{BRAF}+$ and $\mathrm{BRAF}$ - group are approximately equal, it can be assumed that in our cohort the BRAF+ mutation has at the very least no major impact on survival time.

Even if the BRAF-status alone is not associated with tumor recurrence, our results demonstrate the aggressive tumor behavior of BRAF+ PTC. The question arises whether BRAF mutation plays a role as a prognostic marker, and how we should deal with BRAF+ tumors. This issue is not new since many different prognostic scales have been established, among which the AGES, AMES and MACIS scale are the most commonly used (43). They all have in common that the BRAF mutation status is not considered. Due to the inconsistent role of BRAF mutation on the increased risk of relapse, the ATA recommendations do not recommend the routine use of BRAF mutation analysis on initial risk stratification, but they present an additional continuous risk scale including the BRAF status for disease recurrence (6). This shows that risk stratification in thyroid carcinoma should not be dependent on single parameters such as BRAF mutation. It is much more a combination of several factors and "an ongoing process" as outlined by Omry-Orbach et al. (27), which is highlighted by our results. BRAF mutation status could be considered as one component of a basket full of risk factors in the assessment of tumor recurrence for an individualized tailored diagnostic and therapeutic strategy.

Regarding PTC subtypes such as microcarcinoma (pT1a), which might have an indolent prognosis, it is often discussed how BRAF+ microcarcinoma should be handled, i.e. watchful waiting $v s$. hemithyroidectomy or total thyroidectomy and/or prophylactic central node lymphadenectomy. There is also disagreement on this issue as the influence of BRAF V600E mutation is controversial $(36,44-46)$. Due to the retrospective design, the small amount of microcarcinoma, and the postoperative determination of BRAF status in our study, a general recommendation for a more aggressive surgical approach cannot be given. But the potential multifocal growth of $\mathrm{BRAF}+$ tumors should be considered in the decisionmaking process for resection of the contralateral side and lymph node dissection after an initial hemithyroidectomy. This procedure presupposes that BRAF V600E mutation status is routinely performed in cases of postoperatively detected PTC which is not clinically widespread yet.

\section{Conclusions}

Based on our results, more aggressive tumor behavior of BRAF+ PTC in terms of multifocal tumor growth and extrathyroidal expansion may be concluded. However, it is important to note that this tumor behavior was not 
associated with higher tumor relapse in a median follow-up of five years. It seems that BRAF V600E status alone does not influence patient outcome and its routine postoperative determination remains currently without therapeutic consequences, and therefore does not need to be performed routinely. It should however be considered together with further risk factors such lymph node status for an individualized tumor follow-up. The role of BRAF status in papillary microcarcinoma remains debatable, and it should be explored in further studies, for instance with the focus on survival time, as it may possibly change the therapeutic procedure.

\section{Acknowledgments}

We thank Belinda Verdoodt and Rolf-Peter Henke for the assistance in preparation and analysis of the tumor specimen.

Funding: None.

\section{Footnote}

Reporting Checklist: The authors have completed the STROBE reporting checklist. Available at http://dx.doi. org/10.21037/gs-20-244

Data Sharing Statement: Available at http://dx.doi. org/10.21037/gs-20-244

Peer Review File: Available at http://dx.doi.org/10.21037/gs20-244

Conflicts of Interest: All authors have completed the ICMJE uniform disclosure form (available at http://dx.doi. org/10.21037/gs-20-244). The authors have no conflicts of interest to declare.

Ethical Statement: The authors are accountable for all aspects of the work in ensuring that questions related to the accuracy or integrity of any part of the work are appropriately investigated and resolved. The study was conducted in accordance with the Declaration of Helsinki (as revised in 2013). The study was approved by the institutional/regional/national ethics/committee/ethics board of the Carl von University of Oldenburg (No. 005/2017) and informed consent was taken from all the patients.
Open Access Statement: This is an Open Access article distributed in accordance with the Creative Commons Attribution-NonCommercial-NoDerivs 4.0 International License (CC BY-NC-ND 4.0), which permits the noncommercial replication and distribution of the article with the strict proviso that no changes or edits are made and the original work is properly cited (including links to both the formal publication through the relevant DOI and the license). See: https://creativecommons.org/licenses/by-nc-nd/4.0/.

\section{References}

1. Aschebrook-Kilfoy B, Ward MH, Sabra MM, et al. Thyroid cancer incidence patterns in the United States by histologic type, 1992-2006. Thyroid 2011;21:125-34.

2. Lise M, Franceschi S, Buzzoni C, et al. Changes in the incidence of thyroid cancer between 1991 and 2005 in Italy: a geographical analysis. Thyroid 2012;22:27-34.

3. Pereira JA, Jimeno J, Miquel J, et al. Nodal yield, morbidity, and recurrence after central neck dissection for papillary thyroid carcinoma. Surgery 2005;138:1095-100, discussion 1100-1.

4. Wada N, Duh QY, Sugino K, et al. Lymph node metastasis from 259 papillary thyroid microcarcinomas: frequency, pattern of occurrence and recurrence, and optimal strategy for neck dissection. Ann Surg 2003;237:399-407.

5. Mazzaferri EL, Jhiang SM. Long-term impact of initial surgical and medical therapy on papillary and follicular thyroid cancer. Am J Med 1994;97:418-28.

6. Haugen BR. 2015 American Thyroid Association Management Guidelines for Adult Patients with Thyroid Nodules and Differentiated Thyroid Cancer: What is new and what has changed? Cancer 2017;123:372-81.

7. Ito Y, Uruno T, Nakano K, et al. An observation trial without surgical treatment in patients with papillary microcarcinoma of the thyroid. Thyroid 2003;13:381-7.

8. Pacini F, Schlumberger M, Dralle H, et al. European consensus for the management of patients with differentiated thyroid carcinoma of the follicular epithelium. Eur J Endocrinol 2006;154:787-803.

9. Henry JF, Gramatica L, Denizot A, et al. Morbidity of prophylactic lymph node dissection in the central neck area in patients with papillary thyroid carcinoma. Langenbecks Arch Surg 1998;383:167-9.

10. Lee YS, Kim SW, Kim SW, et al. Extent of routine central lymph node dissection with small papillary thyroid carcinoma. World J Surg 2007;31:1954-9. 
11. Hwang HS, Orloff LA. Efficacy of preoperative neck ultrasound in the detection of cervical lymph node metastasis from thyroid cancer. Laryngoscope 2011;121:487-91.

12. Iyer NG, Shaha AR. Central compartment dissection for well differentiated thyroid cancer ... and the band plays on. Curr Opin Otolaryngol Head Neck Surg 2011;19:106-12.

13. Cohen Y, Xing M, Mambo E, et al. BRAF mutation in papillary thyroid carcinoma. J Natl Cancer Inst 2003;95:625-7.

14. Kimura ET, Nikiforova MN, Zhu Z, et al. High prevalence of BRAF mutations in thyroid cancer: genetic evidence for constitutive activation of the RET/PTC-RAS-BRAF signaling pathway in papillary thyroid carcinoma. Cancer Res 2003;63:1454-7.

15. Nikiforova MN, Kimura ET, Gandhi $M$, et al. BRAF mutations in thyroid tumors are restricted to papillary carcinomas and anaplastic or poorly differentiated carcinomas arising from papillary carcinomas. J Clin Endocrinol Metab 2003;88:5399-404.

16. Xing M. BRAF mutation in thyroid cancer. Endocr Relat Cancer 2005;12:245-62.

17. Xing M, Alzahrani AS, Carson KA, et al. Association between BRAF V600E mutation and recurrence of papillary thyroid cancer. J Clin Oncol 2015;33:42-50.

18. Joo JY, Park JY, Yoon YH, et al. Prediction of occult central lymph node metastasis in papillary thyroid carcinoma by preoperative BRAF analysis using fineneedle aspiration biopsy: a prospective study. J Clin Endocrinol Metab 2012;97:3996-4003.

19. Melck AL, Yip L, Carty SE. The utility of BRAF testing in the management of papillary thyroid cancer. Oncologist 2010;15:1285-93.

20. Brzeziańska E, Pastuszak-Lewandoska D, Wojciechowska $\mathrm{K}$, et al. Investigation of V600E BRAF mutation in papillary thyroid carcinoma in the Polish population. Neuro Endocrinol Lett 2007;28:351-9.

21. Fugazzola L, Puxeddu E, Avenia N, et al. Correlation between B-RAFV600E mutation and clinico-pathologic parameters in papillary thyroid carcinoma: data from a multicentric Italian study and review of the literature. Endocr Relat Cancer 2006;13:455-64.

22. Gouveia C, Can NT, Bostrom A, et al. Lack of association of BRAF mutation with negative prognostic indicators in papillary thyroid carcinoma: the University of California, San Francisco, experience. JAMA Otolaryngol Head Neck Surg 2013;139:1164-70.

23. Ito $\mathrm{Y}$, Yoshida $\mathrm{H}$, Maruo R, et al. BRAF mutation in papillary thyroid carcinoma in a Japanese population: its lack of correlation with high-risk clinicopathological features and disease-free survival of patients. Endocr J 2009;56:89-97.

24. Tuttle RM, Haugen B, Perrier ND. Updated American Joint Committee on Cancer/Tumor-Node-Metastasis Staging System for Differentiated and Anaplastic Thyroid Cancer (Eighth Edition): What Changed and Why? Thyroid 2017;27:751-6.

25. Jo YS, Huang S, Kim YJ, et al. Diagnostic value of pyrosequencing for the BRAF V600E mutation in ultrasound-guided fine-needle aspiration biopsy samples of thyroid incidentalomas. Clin Endocrinol (Oxf) 2009;70:139-44.

26. Yeo MK, Liang ZL, Oh T, et al. Pyrosequencing cut-off value identifying BRAFV600E mutation in fine needle aspiration samples of thyroid nodules. Clin Endocrinol (Oxf) 2011;75:555-60.

27. Omry-Orbach G. Risk Stratification in Differentiated Thyroid Cancer: An Ongoing Process. Rambam Maimonides Med J 2016;7:e0003.

28. Barbaro D, Incensati RM, Materazzi G, et al. The BRAF V600E mutation in papillary thyroid cancer with positive or suspected pre-surgical cytological finding is not associated with advanced stages or worse prognosis. Endocrine 2014;45:462-8.

29. Sancisi V, Nicoli D, Ragazzi M, et al. BRAFV600E mutation does not mean distant metastasis in thyroid papillary carcinomas. J Clin Endocrinol Metab 2012;97:E1745-9.

30. Shen X, Zhu G, Liu R, et al. Patient Age-Associated Mortality Risk Is Differentiated by BRAF V600E Status in Papillary Thyroid Cancer. J Clin Oncol 2018;36:438-45.

31. Basolo F, Torregrossa L, Giannini R, et al. Correlation between the BRAF V600E mutation and tumor invasiveness in papillary thyroid carcinomas smaller than 20 millimeters: analysis of 1060 cases. J Clin Endocrinol Metab 2010;95:4197-205.

32. Elisei R, Viola D, Torregrossa L, et al. The BRAF V600E mutation is an independent, poor prognostic factor for the outcome of patients with low-risk intrathyroid papillary thyroid carcinoma: single-institution results from a large cohort study. J Clin Endocrinol Metab 2012;97:4390-8.

33. Frasca F, Nucera C, Pellegriti G, et al. BRAF (V600E) mutation and the biology of papillary thyroid cancer. Endocr Relat Cancer 2008;15:191-205.

34. Kebebew E, Weng J, Bauer J, et al. The prevalence and prognostic value of BRAF mutation in thyroid cancer. Ann 
Surg 2007;246:466.

35. Kim TH, Park YJ, Lim JA, et al. The association of the BRAFV600E mutation with prognostic factors and poor clinical outcome in papillary thyroid cancer. Cancer 2012;118:1764-73.

36. Li F, Chen G, Sheng C, et al. BRAFV600E mutation in papillary thyroid microcarcinoma: a meta-analysis. Endocr Relat Cancer 2015;22:159-68.

37. Lim JY, Hong SW, Lee YS, et al. Clinicopathologic implications of the BRAF V600E mutation in papillary thyroid cancer: a subgroup analysis of 3130 cases in a single center. Thyroid 2013;23:1423-30.

38. Tufano RP, Teixeira GV, Bishop J, et al. BRAF mutation in papillary thyroid cancer and its value in tailoring initial treatment: a systematic review and meta-analysis. Medicine 2012;91:274-86.

39. Xing $M$, Clark D, Guan H, et al. BRAF mutation testing of thyroid fine-needle aspiration biopsy specimens for preoperative risk stratification in papillary thyroid cancer. J Clin Oncol 2009;27:2977-82.

40. Bates MF, Lamas MR, Randle RW, et al. Back so soon? Is early recurrence of papillary thyroid cancer really just persistent disease? Surgery 2018;163:118-23.

41. Elisei R, Schlumberger M, Driedger A, et al. Followup of low-risk differentiated thyroid cancer patients who

Cite this article as: Tabriz N, Grone J, Uslar V, Tannapfel A, Weyhe D. BRAF V600E mutation correlates with aggressive clinico-pathological features but does not influence tumor recurrence in papillary thyroid carcinoma-10-year singlecenter results. Gland Surg 2020;9(6):1902-1913. doi: 10.21037/gs20-244 underwent radioiodine ablation of postsurgical thyroid remnants after either recombinant human thyrotropin or thyroid hormone withdrawal. J Clin Endocrinol Metab 2009;94:4171-9.

42. Niederer-Wüst SM, Jochum W, Forbs D, et al. Impact of clinical risk scores and BRAF V600E mutation status on outcome in papillary thyroid cancer. Surgery 2015;157:119-25.

43. Czarniecka A, Oczko-Wojciechowska M, Barczynski M. BRAF V600E mutation in prognostication of papillary thyroid cancer (PTC) recurrence. Gland Surg 2016;5:495-505.

44. Kim KJ, Kim SG, Tan J, et al. BRAF V600E status may facilitate decision-making on active surveillance of lowrisk papillary thyroid microcarcinoma. Eur J Cancer 2020;124:161-9.

45. Park VY, Kim EK, Lee HS, et al. Real-Time PCR Cycle Threshold Values for the BRAFV600E Mutation in Papillary Thyroid Microcarcinoma May Be Associated With Central Lymph Node Metastasis: A Retrospective Study. Medicine 2015;94:e1149.

46. Tallini G, de Biase D, Durante C, et al. BRAF V600E and risk stratification of thyroid microcarcinoma: a multicenter pathological and clinical study. Mod Pathol 2015;28:1343-59. 